\title{
Crecimiento y calidad de plántulas de café (Coffea arabica) injertadas sobre Coffea canephora
}

\author{
frente a nematodos en vivero
}

\section{Growth and quality of coffee cultivars seedling (Coffea arabica) grafted onto Coffea canephora \\ facing nematodes in greenhouse}

\author{
Borjas-Ventura Ricardo*, Andía Alarcón Eduard, Alarcón-Águila Gerson, Estelita Castro Silvia, \\ Julca-Otiniano Alberto
}

\begin{tabular}{l}
\hline Datos del Articulo \\
\hline Facultad de Agronomía. Departamento de \\
Fitotecnia. Universidad Nacional Agraria \\
La Molina. Av. La Molina s/n La Molina. \\
Lima, Perú. \\
Teléfono: +51998174923 / 016147800. \\
*Dirección de contacto: \\
Ricardo Borjas-Ventura \\
Facultad de Agronomía. Departamento de \\
Fitotecnia. Universidad Nacional Agraria \\
La Molina. Av. La Molina s/n La Molina. \\
Lima, Perú. \\
Teléfono: +51998174923 / 016147800 \\
E-mail: rborjas@ @lamolina.edu.pe \\
\hline
\end{tabular}

\section{Palabras clave:}

Meloidogyne,

crecimiento,

biomasa,

indice de robustez,

indice de calidad de Dickson.

\section{Resumen}

El uso de plantas injertadas es una técnica usada para el control de Meloidogyne spp. en café, incluye múltiples factores que influyen sobre el comportamiento de la púa y el porta-injerto, también sobre el crecimiento y desarrollo de la planta injertada. En Perú, son pocos los trabajos realizados sobre esta relación, de manera especial el caso del café. Este experimento se realizó con el objetivo de evaluar el comportamiento de nueve cultivares de café: Laurina IAC 870, Catuaí vermelho IAC 144, Catuaí vermelho IAC 99, Catuaí amarelo IAC 86, Caturra amarelo Col. 88 IAC 476, Bourbon vermelho IAC 662, Caturra vermelho IAC 477, Obatã IAC 1669-20 y Limaní, injertados sobre Coffea canephora en presencia y ausencia de Meloidogyne spp. en fase de vivero. Los resultados mostraron que en altura y diámetro de tallo destacó el injerto Obatã IAC 1669-20/Robusta (inoculado), mientras que para número de hojas, el injerto Caturra Vermelho IAC 477/Robusta (inoculado). Al medir la biomasa seca de la parte aérea y radicular, los injertos Laurina IAC 870/Robusta (no inoculado), Caturra Vermelho IAC 477/Robusta (inoculado) y Obatã IAC 1669-20/Robusta (inoculado) tuvieron los valores más altos. Para el Índice de Calidad de Dickson (ICD), los injertos Caturra Vermelho IAC 477/Robusta (inoculado) y Obatã IAC 1669-20/Robusta (inoculado) fueron los más destacados. Se concluyó que Caturra Vermelho IAC 477 y Obatã IAC 1669-20 injertados sobre $C$. canephora fueron los cultivares que mejor respondieron ante la presencia de Meloidogyne spp.

() 2018. Journal of the Selva Andina Biosph. Bolivia. Todos los derechos reservados.

\section{Abstract}

J Selva Andina Biosph. 2018; 6(2):28-41.

\section{Historial del artículo. \\ Recibido marzo, 2018 Devuelto mayo 2018 \\ Aceptado septiembre, 2018 . \\ Disponible en línea, noviembre 2018. \\ Editado por: \\ Selva Andina \\ Research Society}

\section{Key words:}

\section{Meloidogyne,}

growth,

biomass,

robustness index,

Dickson quality index.
The use of grafted plants is a technique used for the control of Meloidogyne spp. in coffee, which includes multiple factors that influence the behavior of the barb and the graft holder and also the growth and development of the grafted plant. In Peru, little work has been done to know the relationship between the barb and the graft holder, especially in the case of coffee. This experiment was carried out with the objective of evaluating the behavior of nine coffee cultivars: Laurina IAC 870, Catuaí vermelho IAC 144, Catuaí vermelho IAC 99, Catuaí amarelo IAC 86, Caturra amarelo Col. 88 IAC 476, Bourbon vermelho IAC 662, Caturra vermelho IAC 477, Obatã IAC 1669-20 and Limaní, grafted on Coffea canephora in presence and absence of Meloidogyne spp. in nursery phase. The results showed that the Obatã IAC 166920/Robusta graft (inoculated) stood out in height and stem diameter, while for the number of leaves was the Caturra Vermelho IAC 477/Robusta graft (inoculated). When measuring the dry biomass of the aerial and root part, the Laurina IAC 870/Robusta (not inoculated), Caturra Vermelho IAC 477/Robusta (inoculated) and Obatã IAC 1669-20/Robusta (inoculated) grafts had the highest values. For the Dickson Quality Index (ICD), the Caturra Vermelho IAC 477/Robusta (inoculated) and Obatã IAC 1669-20/Robusta (inoculated) grafts were the most outstanding. It was concluded that Caturra Vermelho IAC 477 and Obatã IAC 1669-20 grafted on $C$. canephora were the cultivars that best responded to the presence of Meloidogyne spp. 


\section{Introducción}

El café es uno de los productos agrícolas más importantes de la economía peruana, de hecho, para el 2017, su exportación generó ingresos por 626 millones de dólares ${ }^{1}$, además, de ser una fuente de empleo para 2 millones de personas, reflejando así, la importancia social de esta actividad agrícola. Desde una perspectiva global, Perú es el séptimo productor de café y el segundo agroexportador de café orgánico. ${ }^{2}$

A pesar de la importancia económica y social del café, aún existen muchos desafíos por superar, entre ellos, el control de nematodos fitoparásitos como los del género Meloidogyne spp., especies que se encuentran distribuidas en todos los países productores de café, incluyendo el Perú ${ }^{3}$, causando una disminución de la producción entre $60-90 \% .^{4,5}$

Una de las formas de manejo de estos fitopatógenos es mediante el uso de injertos. ${ }^{6,7}$ En café, se recomienda el uso de Coffea canephora, cuya característica es el vigor y la resistencia a nematodos. ${ }^{5,8-10}$

Además de las características mencionadas, C. canephora (como porta-injerto) puede ejercer influencia, sobre el injerto, en aspectos fisiológicos (intercambio de gases, transpiración y asimilación neta de carbono) y de crecimiento. ${ }^{11,12}$

Por otra parte, la comunicación entre el porta-injerto y la yema (injerto), se ve afectado por factores externos como la presencia de nematodos, los que pueden favorecer o perjudicar el crecimiento del injerto en función de su cantidad ${ }^{13-16} \mathrm{y}$ de los injertos usados. ${ }^{17}$

A pesar de la información existente sobre el comportamiento de los injertos usando como portainjerto $C$. canephora en presencia y ausencia de nematodos ${ }^{17}$, en el Perú existen pocos estudios referidos a este tema, así por ejemplo, Julca et $a l^{18}$ re 29 portaron una mejor respuesta, ante la presencia de nematodos, en plantas injertadas de Icatú vermelho y Caturra vermelho, sobre café Robusta. Además, es importante resaltar la importancia de estudios locales, ya que el comportamiento de plantas francas, como injertadas se encuentran fuertemente relacionadas a factores agroclimáticos.

Otro de los principales problemas en la caficultura peruana, que se refleja en la baja productividad de este cultivo, es la falta de material genético mejorado, ante esta situación, Julca et al. ${ }^{19}$ introdujeron variedades de café mejoradas desde Brasil. Pero no se conoce el comportamiento de las variedades introducidas cuando son injertadas sobre $C$. canepho$r a$, especialmente frente a nematodos del género Meloidogyne. En este contexto, se realizó esta investigación con el objetivo de cuantificar el crecimiento y la calidad de las plántulas de diferentes cultivares de café injertadas sobre café Robusta en presencia de nematodos del género Meloidogyne spp., en fase de vivero.

\section{Materiales y métodos}

El experimento fue conducido en el "Fundo La Génova" de la Universidad Nacional Agraria La Molina (UNALM), tuvo una duración de 5 meses de febrero a julio 2015. Las coordenadas del campo experimental son $11^{\circ} 00^{\prime}-11^{\circ} 15^{\prime} \mathrm{S}$ y $75^{\circ} 15^{\prime}-75^{\circ} 30^{\prime}$ O, en el distrito de San Ramón, Junín-Perú (Figura 1). La zona se caracteriza por presentar un clima tropical con temperatura de $17-31{ }^{\circ} \mathrm{C}$, se encuentra a una altura de 965 msnm. ${ }^{20}$

Las semillas del porta-injerto (C. canephora) y de los injertos (Laurina IAC 870, Catuaí Vermelho IAC 144, Catuaí Vermelho IAC 99, Catuaí Amarelo 
IAC 86, Caturra Amarelo Col. 88 IAC 476, Bourbon Vermelho IAC 662, Caturra Vermelho IAC 477, Obatã IAC 1669-20 y Limaní) fueron obteni- das del banco de germoplasma de la UNALM. Todos los cultivares, excepto Limaní, fueron introducidos desde Brasil.

Figura 1 Ubicación del distrito de San Ramón (Departamento de Junín), selva central del Perú

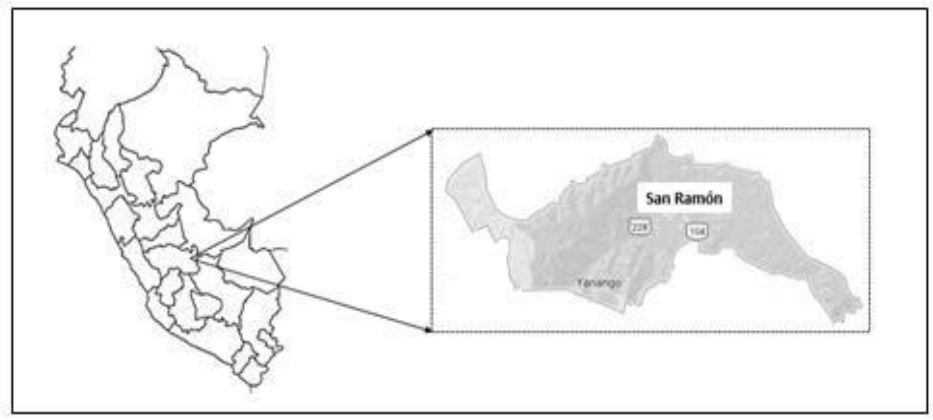

Las semillas fueron sembradas en camas hechas con arena de río, cada cama tuvo $1 \mathrm{~m}$ de ancho, $0.30 \mathrm{~m}$ de alto y $1 \mathrm{~m}$ de largo, luego fueron cubiertas con una capa de arena de 0.5-1 cm de espesor, 75 días después de la siembra se realizó el injerto hipocoti- ledonal. ${ }^{21}$ En ese momento, el porta-injerto tenia las hojas cotiledonales completamente desarrolladas, plantas como injertos aún se mantenían en estado de 'palito de fósforo.' 18

Tabla 1 Características químicas del substrato usado para el ensayo de cultivares de café arábicas injertados sobre $C$. canephora

\begin{tabular}{lccc}
\hline \multicolumn{1}{c}{ Caracteristicas } & \multicolumn{2}{c}{ Caracteristicas } \\
\hline $\mathrm{pH}$ & 5.96 & Potasio $(\mathrm{ppm})$ & 111 \\
$\mathrm{CE}(\mathrm{Ds} / \mathrm{m})$ & 0.34 & $\mathrm{CIC}\left(\mathrm{cmol}_{(+)} \mathrm{kg}^{-1}\right)$ & 15.68 \\
$\mathrm{CaCO}_{3}(\%)$ & 0 & Suma de cationes & 12.74 \\
$\mathrm{MO}(\%)$ & 2.6 & Suma de bases & 12.64 \\
Fósforo $(\mathrm{ppm})$ & 24.3 & \% Saturación de bases & 81 \\
\hline CIC: Capacidad de intercambio cationico; CE: Conductividad electrica; MO: Materia orgánica
\end{tabular}

Cada planta injertada se colocó en bolsas negras de polietileno (5" x 7" x 1.5") con capacidad de $0.5 \mathrm{~kg}$. El sustrato, fue tierra de una finca cafetalera, que previamente se desinfecto con tratamiento térmico $\left(80{ }^{\circ} \mathrm{C} / 1 \mathrm{~h}\right)$. Las características químicas del substrato están descritas en Tabla 1. Una vez colocadas en las bolsas, las plantas fueron llevadas a un tinglado, y permanecieron durante todo el experimento. Las plántulas de café fueron regadas hasta la capacidad de campo. Las plagas y malezas fueron monitoreadas con el objetivo de asegurar su control.

Como inoculo de nematodos se utilizó huevos+juveniles del segundo estadio (J2) (10000/kg substrato), fueron obtenidos de raíces de café con síntomas de agallas. ${ }^{13}$

Los tratamientos estuvieron formados por cultivares Laurina IAC 870, Catuaí Vermelho IAC 144, Catuaí Vermelho IAC 99, Catuaí Amarelo IAC 86, Caturra 
Amarelo Col. 88 IAC 476, Bourbon Vermelho IAC 662, Caturra Vermelho IAC 477, Obatã IAC 1669-

20 y Limaní, todos injertados sobre el cultivar Ro- busta, en presencia y ausencia de nematodos, un total de 18 tratamientos con 10 repeticiones (Tabla 2).

Tabla 2 Tratamientos usados en el ensayo de cultivares de café arábicas injertados sobre Coffea canephora

\begin{tabular}{clcclcr}
\hline Tratamientos & \multicolumn{1}{c}{ Descripción } & Inoculado & Tratamientos & \multicolumn{1}{c}{ Descripción } & Inoculado \\
\hline T1 & Laurina IAC 870/Robusta & SI & T10 & Caturra Amarelo Col. 8 IAC & 476/Robusta \\
T2 & Laurina IAC 870/Robusta & NO & T11 & Bourbon Vermelho IAC 662/Robusta & SI \\
T3 & $\begin{array}{l}\text { Catuaí Vermelho IAC } \\
\text { 144/Robusta }\end{array}$ & SI & T12 & Bourbon Vermelho IAC 662/Robusta & NO \\
T4 & $\begin{array}{l}\text { Catuaí Vermelho IAC } \\
\text { 144/Robusta }\end{array}$ & NO & T13 & Caturra Vermelho IAC 477/Robusta & SI \\
T5 & $\begin{array}{l}\text { Catuaí Vermelho IAC } \\
\text { 99/Robusta }\end{array}$ & SI & T14 & Caturra Vermelho IAC 477/Robusta & NO \\
T6 & $\begin{array}{l}\text { Catuaí Vermelho IAC } \\
\text { 99/Robusta }\end{array}$ & NO & T15 & Obatã IAC 1669-20/Robusta & SI \\
T7 & $\begin{array}{l}\text { Catuaí Amarelo IAC } \\
\text { 86/Robusta }\end{array}$ & SI & T16 & Obatã IAC 1669-20/Robusta & NO \\
T8 & $\begin{array}{l}\text { Catuaí Amarelo IAC } \\
\text { 86/Robusta }\end{array}$ & NO & T17 & Limaní/Robusta & SI \\
T9 & Caturra Amarelo Col. 8 IAC & SI & T18 & Limaní/Robusta & NO \\
\hline
\end{tabular}

Las evaluaciones se realizaron mensualmente desde el momento que se aplicaron los tratamientos. Se cuantificó altura de la planta $(\mathrm{AP})(\mathrm{cm})$, diámetro del tallo (DDT) (mm), número de hojas $(\mathrm{NH})$, biomasa seca (BS) (g), Índice de Robustez (IR), relación entre el peso seco aéreo y el peso seco de la raíz (A/R) y el Índice de Calidad de Dickson (ICD), siendo este último, un buen indicador de la calidad de plántulas de café en vivero. ${ }^{22}$

La AP se midió desde la base del tallo hasta la yema apical, para tal fin se usó una regla graduada. Con la ayuda de un vernier se calculó el DDT, se midió un centímetro encima de la zona del injerto. El NH fue determinado mediante su conteo en cada evaluación. Para $\mathrm{A} / \mathrm{R}$, los tejidos fueron sometidos a $75^{\circ} \mathrm{C}$ por $48 \mathrm{~h}$. El IR se calculó usando la fórmula: $\mathrm{AP} / \mathrm{DDT}^{23}$, mientras que, para el ICD se usó la fórmula PST/[(IR $)+(\mathrm{A} / \mathrm{R})]^{24}$, donde el PST representa el peso seco total.

El diseño experimental fue completamente al azar (DCA) con un arreglo factorial $9 \times 2$ (9 cultivares $\mathrm{x}$ 31
2 niveles de Meloidogyne). Todos los datos fueron sometidos a un análisis de variancia (ANOVA) y comparados mediante la prueba de Tukey (95\%) con el software AGROSTAT ${ }^{4}$, además se determinó la ecuación de regresión de los resultados más importante. Asimismo, se realizó un agrupamiento de los tratamientos. Los datos que no cumplieron con la distribución normal fueron transformados de acuerdo con Montgomery ${ }^{25}$ (Tabla 3).

Tabla 3 Transformación de datos con el método BoxCox, de las variables que no presentaron distribución normal

\begin{tabular}{lc}
\hline \multicolumn{1}{c}{ Variable } & Transformación \\
\hline Altura de planta (0 ddt) & $\left(\mathrm{Y}^{-0.5}-1\right) /-0.5$ \\
Diámetro de tallo (30 ddt) & $\left(\mathrm{Y}^{-0.5}-1\right) /-0.5$ \\
Diámetro de tallo (60 ddt) & $\left(\mathrm{Y}^{-0.5}-1\right) /-0.5$ \\
Diámetro de tallo (90 ddt) & $\left(\mathrm{Y}^{-2}-1\right) /-2$ \\
Número de hojas (30 ddt) & $\left(\mathrm{Y}^{1.5}-1\right) / 1.5$ \\
Peso seco aéreo & $\mathrm{Y}^{0.15}$ \\
Peso seco raíz & $\mathrm{Y}^{0.15}$ \\
ICD & $\mathrm{Y}^{0.15}$ \\
\hline ddt: días después de trasplante, ICD: Índice de Calidad de Dickson
\end{tabular}




\section{Resultados}

Como se puede observar, Tabla 4, luego del análisis factorial, se notó que la interacción entre nematodos y cultivares afectaron todas las variables, con excepción del NH y A/R. En el caso del NH, ésta fue afectada por el factor cultivar.

Además de la interacción entre los factores, también se cuantificó el crecimiento de los injertos. En primer lugar, se evaluó la AP, observándose que los injertos Laurina IAC 870/Robusta (no inoculado),
Bourbon vermelho IAC 662/Robusta (inoculado) y Obatã IAC 1669-20/Robusta (inoculado) fueron estadísticamente más altos con 13.79, 13.12 y 13.89 $\mathrm{cm}$ respectivamente, mientras que las plantas con menor altura fueron los injertos Catuaí amarelo IAC86/Robusta (no inoculado) y Caturra vermelho IAC 477 (no inoculado) con 7.56 y $7.75 \mathrm{~cm}$ respectivamente (Figura 2).

Tabla 4 Cuadrados medios de los factores principales (Cultivar y presencia de Meloidogyne) y la interacción de ambos factores en el ensayo de cultivares de café arábicas injertados sobre $C$. canephora

\begin{tabular}{cccc}
\hline Variables & \multicolumn{3}{c}{ Factores } \\
\cline { 2 - 4 } & Cultivar & Meloidogyne & Cultivar x Meloidogyne \\
\hline Altura de planta & $72.67^{* *}$ & $57.95^{* *}$ & $14.04^{*}$ \\
Diámetro de tallo & $0.0026^{* *}$ & $0.0067^{* *}$ & $0.0012^{* *}$ \\
Número de hojas & $18.64^{* *}$ & $6.94^{\mathrm{ns}}$ & $4.43^{\mathrm{ns}}$ \\
Peso seco aéreo & $0.355^{* *}$ & $0.165^{* *}$ & $0.537^{* *}$ \\
Peso seco raíz & $0.0156^{* *}$ & $0.0161^{*}$ & $0.0097^{* *}$ \\
A/R & $0.0051^{\mathrm{ns}}$ & $0.0026^{\mathrm{ns}}$ & $0.0063^{\mathrm{ns}}$ \\
IR & $11.86^{* *}$ & $2.03^{*}$ & $1.136^{*}$ \\
ICD & $0.007^{* *}$ & $0.0071^{*}$ & $0.0042^{*}$ \\
\hline A/R: Relación peso seco aéreo/raíz, IR: Índice de Robustez, ICD: Índice de Calidad de Dickson, **Significativo al p $\leq 0.01$, &
\end{tabular}

En el DDT (Figura 2), se notó que hubo un crecimiento con el transcurrir del tiempo, los injertos Catuaí vermelho IAC 144/Robusta (inoculado) y Obatã IAC 1669-20/Robusta (inoculado), fueron significativamente superiores al tratamiento Caturra vermelho IAC 477 (no inoculado) en $31 \%$ en ambos casos.

Para el NH (Figura 2), se notó que Caturra vermelho IAC 477 (inoculado) fue significativamente superior a Catuaí amarelo IAC 86 (no inoculado) en $48 \%$.

Ecuaciones de regresión fueron determinadas en este ensayo (Tabla 5). La dinámica del aumento AP, para Laurina IAC 870/Robusta (no inoculado), Obatã IAC 1669-20/Robusta (inoculado) y Bourbon vermelho IAC 662/Robusta (inoculado), estuvo representada por ecuaciones de primer grado con un
$\mathrm{R}^{2}$ de $97-99 \%$, mientras que Catuaí amarelo IAC 86/Robusta (no inoculado) y Caturra vermelho IAC 477/Robusta (no inoculado) dieron dinámicas de ecuaciones de crecimiento de segundo y tercer grado.

Las ecuaciones de regresión que mejor representan el aumento del DDT fueron de primer y segundo grado para los injertos Catuaí vermelho IAC 144/Robusta y Obatã IAC 1669-20/Robusta (inoculado), en cuanto que, una ecuación de tercer grado dio una mejor correlación para el injerto Caturra vermelho IAC 477/Robusta (no inoculado) (Tabla 5).

El aumento $\mathrm{NH}$, el injerto Caturra vermelho IAC 86/Robusta (inoculado), reporto una ecuación de segundo grado, con menor $\mathrm{NH}$, Catuaí amarelo IAC 
86/Robusta (no inoculado) y Lamaní/Robusta (inogrado respectivamente (Tabla 5).

culado) tuvieron ecuaciones de segundo y primer

Figura 2 Características morfológicas de diferentes cultivares de café injertado sobre Robusta en diferentes momentos de evaluación. A: Altura de la planta. B: Diámetro del tallo. C: Número de hojas. Tukey (p \).05)

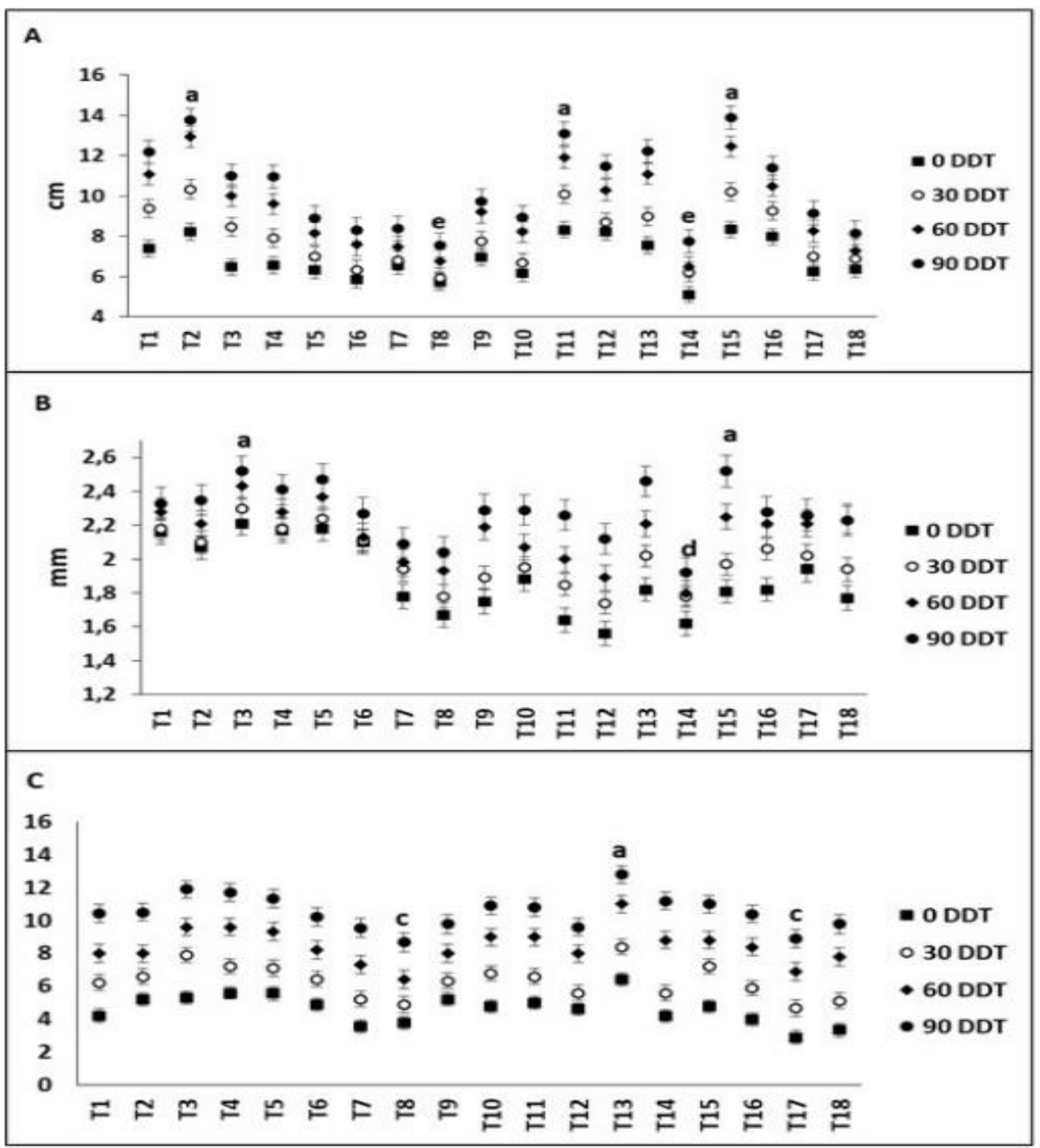

T1: Laurina IAC 870 (inoculado); T2: Laurina IAC 870 (no inoculado); T3: Catuaí Vermelho IAC 144 (inoculado); T4: Catuaí Vermelho IAC 144 (no inoculado); T5: Catuaí Vermelho IAC 99 (inoculado); T6: Catuaí Vermelho IAC 99 (no inoculado); T7: Catuaí Amarelo IAC 86 (inoculado); T8: Catuaí Amarelo IAC 86 (no inoculado); T9: Caturra Amarelo Col. 8 IAC 476 (inoculado); T10: Caturra Amarelo Col. 8 IAC 476 (no inoculado); T11: Bourbon Vermelho IAC 662 (inoculado); T12: Bourbon Vermelho IAC 662 (no inoculado); T13: Caturra Vermelho IAC 477 (inoculado); T14: Caturra Vermelho IAC 477 (no inoculado); T15: Obatã IAC 1669-20 (inoculado); T16: Obatã IAC 1669-20 (no inoculado); T17: Limaní (inoculado); T18: Limaní (no inoculado). DDT: Días después del trasplante. 
Tabla 5 Ecuaciones de regresión de altura de la planta, diámetro del tallo y número de hojas por momento de evaluación en el ensayo de cultivares de café arábicas injertados sobre $C$. canephora

\begin{tabular}{cccc}
\hline Tratamiento & Ecuación & $\mathbf{r}^{\mathbf{2}}$ & $\mathbf{V}$ \\
\hline Laurina IAC 870/Robusta (no inoculado) & $\mathrm{Y}=1.93 \mathrm{X}+6.51$ & 0.97 & $\mathrm{AP}+$ \\
Obatã IAC 1669-20/Robusta (inoculado) & $\mathrm{Y}=1.89 \mathrm{X}+6.50$ & 0.99 & $\mathrm{AP}+$ \\
Bourbon Vermelho IAC 662/Robusta (inoculado) & $\mathrm{Y}=1.62 \mathrm{X}+6.81$ & 0.99 & $\mathrm{AP}+$ \\
Catuaí Amarelo IAC 86/Robusta (no inoculado) & $\mathrm{Y}=0.145 \mathrm{X}^{2}-0.095 \mathrm{X}+5.66$ & 0.99 & $\mathrm{AP}-$ \\
Caturra Vermelho IAC 477/Robusta (no inoculado) & $\mathrm{Y}=0.3183 \mathrm{X}^{3}-2.345 \mathrm{X}^{2}+5.9167 \mathrm{X}+1.23$ & 1 & $\mathrm{AP}-$ \\
Catuaí Vermelho IAC 144/Robusta (inoculado) & $\mathrm{Y}=0.106 \mathrm{X}+2.10$ & 0.99 & $\mathrm{DT}+$ \\
Obatã IAC 1669-20/Robusta (inoculado) & $\mathrm{Y}=0.0275 \mathrm{X}^{2}+0.1035 \mathrm{X}+1.67$ & 1 & $\mathrm{DT}+$ \\
Caturra Vermelho IAC 477/Robusta (no inoculado) & $\mathrm{Y}=0.04 \mathrm{X}^{3}-0.31 \mathrm{X}^{2}+0.81 \mathrm{X}+1.08$ & 1 & $\mathrm{DT}-$ \\
Caturra Vermelho IAC 477/Robusta (inoculado) & $\mathrm{Y}=2.18 \mathrm{X}+4.20$ & 1 & $\mathrm{NH}+$ \\
Catuaí Amarelo IAC 86/Robusta (no inoculado) & $\mathrm{Y}=0.28 \mathrm{X}^{2}+0.222 \mathrm{X}+3.29$ & 1 & $\mathrm{NH}-$ \\
Limaní/Robusta (inoculado) & $\mathrm{Y}=2.0022 \mathrm{X}+0.78$ & 1 & $\mathrm{NH}-$ \\
\hline
\end{tabular}

V: Variable, AP+: Mayor altura de plantas, AP-: Menos altura de plantas, DT+: Mayor diámetro de tallo, DT-: Menor diámetro de tallo, $\mathrm{NH}+$ : Mayor número de hojas, NH-: Menor número de hojas.

Figura 3 Comparación de medias (Tukey 95\%) del peso seco parte aérea (A), peso seco de raíz (A) y relación peso seco parte aérea/Raíz (B) en el ensayo de cultivares de café arábicas injertados sobre $C$. canephora

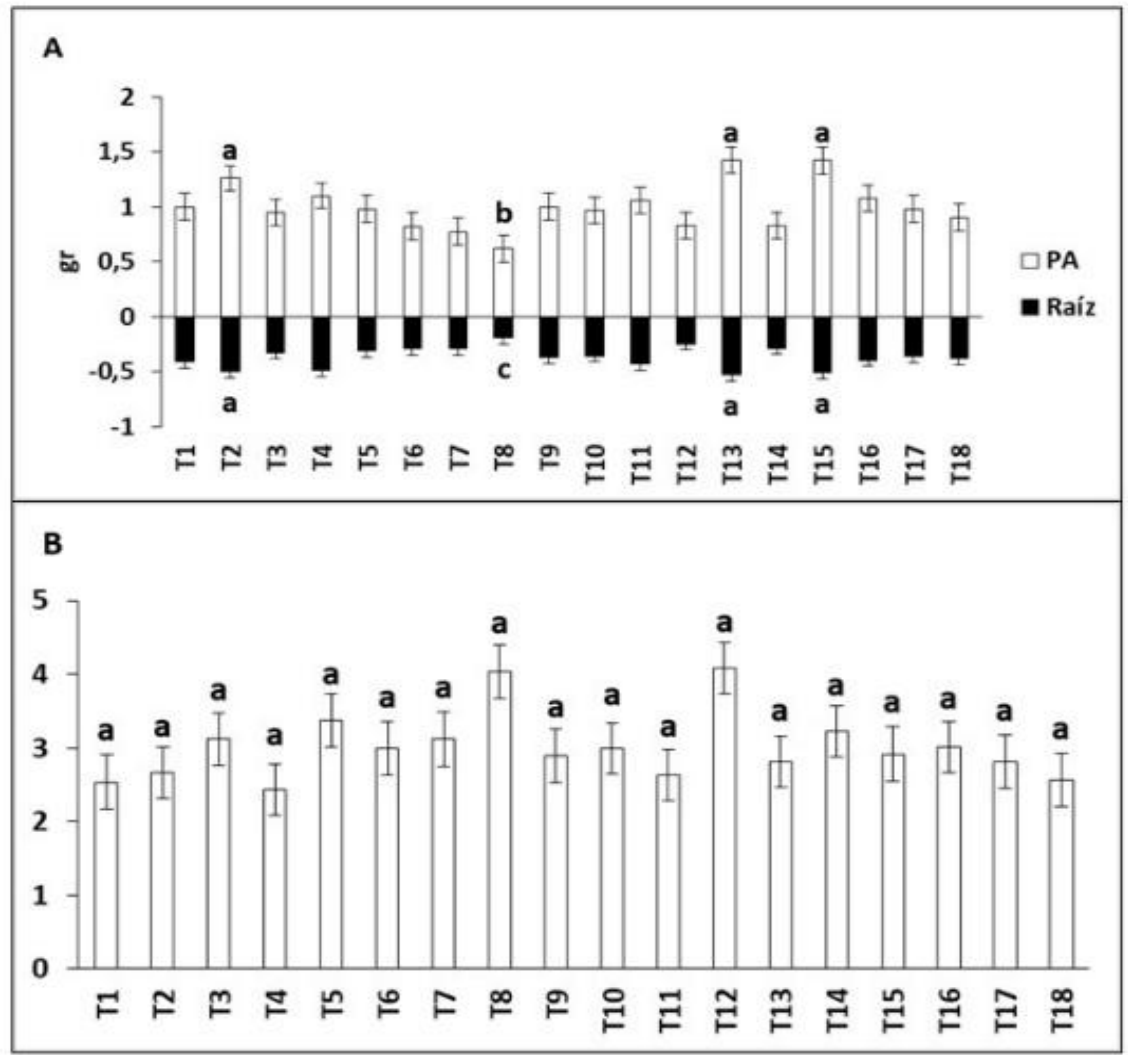

T1: Laurina IAC 870 (inoculado); T2: Laurina IAC 870 (no inoculado); T3: Catuaí Vermelho IAC 144 (inoculado); T4: Catuaí Vermelho IAC 144 (no inoculado); T5: Catuaí Vermelho IAC 99 (inoculado); T6: Catuaí Vermelho IAC 99 (no inoculado); T7: Catuaí Amarelo IAC 86 (inoculado); T8: Catuaí Amarelo IAC 86 (no inoculado); T9: Caturra Amarelo Col. 8 IAC 476 (inoculado); T10: Caturra Amarelo Col. 8 IAC 476 (no inoculado); T11: Bourbon Vermelho IAC 662 (inoculado); T12: Bourbon Vermelho IAC 662 (no inoculado); T13: Caturra Vermelho IAC 477 (inoculado); T14: Caturra Vermelho IAC 477 (no inoculado); T15: Obatã IAC 1669-20 (inoculado); T16: Obatã IAC 1669-20 (no inoculado); T17: Limaní (inoculado); T18: Limaní (no inoculado). PA: Parte aérea. 
Figura 4 Comparación de medias (Tukey 95\%) del Índice de Robustez (A) e Índice de Calidad de Dickson (B) en plantas de café arábicas injertados sobre $C$. canephora

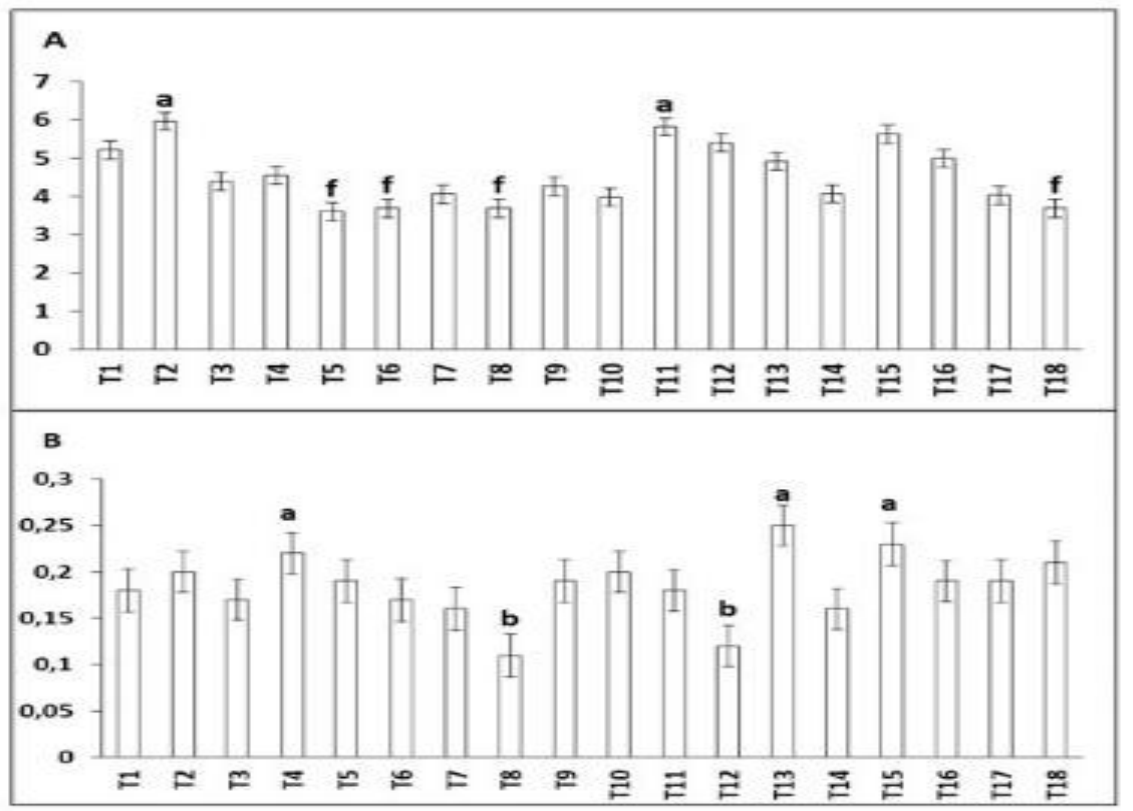

T1: Laurina IAC 870 (inoculado); T2: Laurina IAC 870 (no inoculado); T3: Catuaí Vermelho IAC 144 (inoculado); T4: Catuaí Vermelho IAC 144 (no inoculado); T5: Catuaí Vermelho IAC 99 (inoculado); T6: Catuaí Vermelho IAC 99 (no inoculado); T7: Catuaí Amarelo IAC 86 (inoculado); T8: Catuaí Amarelo IAC 86 (no inoculado); T9: Caturra Amarelo Col. 8 IAC 476 (inoculado); T10: Caturra Amarelo Col. 8 IAC 476 (no inoculado); T11: Bourbon Vermelho IAC 662 (inoculado); T12: Bourbon Vermelho IAC 662 (no inoculado); T13: Caturra Vermelho IAC 477 (inoculado); T14: Caturra Vermelho IAC 477 (no inoculado); T15: Obatã IAC 1669-20 (inoculado); T16: Obatã IAC 1669-20 (no inoculado); T17: Limaní (inoculado); T18: Limaní (no inoculado).

El crecimiento de la BS de los injertos de café (Figura 3). De acuerdo con los resultados, la BS de la parte aérea, Laurina IAC 870/Robusta (no inoculado), Caturra vermelho IAC 477/Robusta (inoculado) y Obatã IAC 1669-20/Robusta (inoculado), fueron significativamente más elevadas que, Catuaí amarelo IAC 86/Robusta (no inoculado) en $103 \%$ (para Laurina IAC 870) y $129 \%$ (para Caturra vermelho IAC 477 y Obatã IAC 1669-20).

En relación BS de raíz, se notó la misma tendencia que el peso seco de la parte aérea (Figura 3). Los injertos Laurina IAC 870/Robusta (no inoculado), Caturra vermelho IAC 477/Robusta (inoculado) y Obatã IAC 1669-20/Robusta (inoculado), fueron significativamente más altos que el injerto Catuaí amarelo IAC 86/Robusta (no inoculado) en $163 \%$, $179 \%$ y $168 \%$ respectivamente.

El IR (Figura 5), la comparación por el test de Tukey (0.05) señala Laurina IAC 870/Robusta (no inoculado) y Bourbon vermelho IAC 662/Robusta (inoculado) fueron los más altos con 5.96 y 5.82 respectivamente. Asimismo, los menores valores fueron para Catuaí vermelho IAC 99/Robusta (inoculado), Catuaí vermelho IAC 99/Robusta (no inoculado), Catuaí amarelo IAC 86/Robusta (no inoculado) y Limaní/Robusta (no inoculado) con 3.6, $3.69,3.69$ y 3.69 respectivamente.

Para ICD, los más destacados fueron Catuaí vermelho IAC 144/Robusta (no inoculado), Caturra vermelho IAC 477/Robusta (inoculado) y Obatã IAC 1669-20/Robusta (inoculado) con valores de 
$0.22,0.25$ y 0.23 . Los valores con menor ICD fueron, Catuaí amarelo IAC 86/Robusta (no inoculado) y Bourbon vermelho IAC 662/Robusta (no inoculado) con 0.11 y 0.12 (Figura 4).

Cuando se realizó el agrupamiento de los tratamientos por el método Ward, notamos, de forma general, la formación de dos grupos. El primer grupo formado por nueve tratamientos, de ellos, tres fueron inoculados y un segundo grupo, de los que tres no inoculados (Figura 5).

\section{Figura 5 Análisis cluster, con el método Ward, en plantas de café arábicas injertados sobre $C$. canephora}

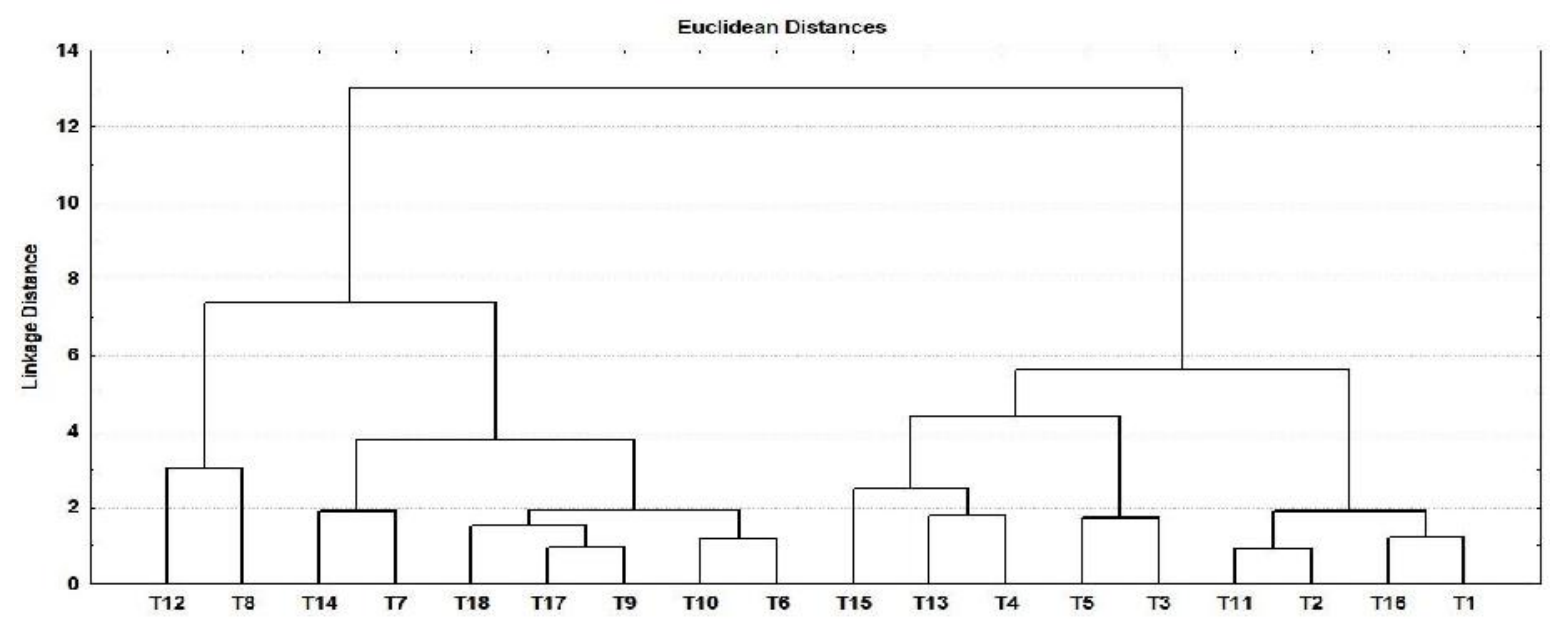

T1: Laurina IAC 870 (inoculado); T2: Laurina IAC 870 (no inoculado); T3: Catuaí Vermelho IAC 144 (inoculado); T4: Catuaí Vermelho IAC 144 (no inoculado); T5: Catuaí Vermelho IAC 99 (inoculado); T6: Catuaí Vermelho IAC 99 (no inoculado); T7: Catuaí Amarelo IAC 86 (inoculado); T8: Catuaí Amarelo IAC 86 (no inoculado); T9: Caturra Amarelo Col. 8 IAC 476 (inoculado); T10: Caturra Amarelo Col. 8 IAC 476 (no inoculado); T11: Bourbon Vermelho IAC 662 (inoculado); T12: Bourbon Vermelho IAC 662 (no inoculado); T13: Caturra Vermelho IAC 477 (inoculado); T14: Caturra Vermelho IAC 477 (no inoculado); T15: Obatã IAC 1669-20 (inoculado); T16: Obatã IAC 1669-20 (no inoculado); T17: Limaní (inoculado); T18: Limaní (no inoculado).

\section{Discusión}

Se estimó el crecimiento y la calidad de plántulas (con el Índice de Calidad de Dickson) de los cultivares de café injertados sobre el café cultivar Robusta, en presencia y ausencia de Meloidogyne spp., estos nematodos son capaces de infectar diferentes especies de plantas, provocando disminución en su rendimiento..$^{26,27}$

Una de las variables evaluadas, AP (Figura 2) reportó un aumento con el transcurrir del tiempo, aunque, los promedios alcanzados no fueron los mismos para todos los tratamientos, de hecho, en su última evaluación, Laurina IAC 870/Robusta (no inoculado), Bourbon Vermelho IAC 662/Robusta (inocula- do) y Obatã IAC 1669-20/Robusta (inoculado) fueron significativamente más altos en comparación con los demás, Catuaí Amarelo IAC 86/Robusta (no inoculado) y Caturra Vermelho IAC 477/Robusta (no inoculado) dieron valores significativamente más bajos (Figura 2).

Laurina IAC 870/Robusta (no inoculado) y Bourbon Vermelho IAC 662/Robusta (inoculado) tuvieron valores similares para AP. Si tomamos en cuenta que Laurina IAC 870 es considerada un cultivar de porte bajo, en tanto que Bourbon Vermelho IAC 662 es considerado de porte alto, podemos inferir que existe una interacción positiva entre el portainjerto Robusta y el cultivar Laurina IAC 870 en ausencia de nematodos. 
En este sentido, investigadores han reportado la existencia de una interacción positiva entre la yema y el porta-injerto, de forma que el crecimiento y desarrollo del injerto se vea favorecido por el portainjerto ${ }^{28,29}$

Obatã IAC 1669-20, otro cultivar de porte bajo, que injertado sobre Robusta, en presencia de nematodos logro la misma altura que Bourbon amárelo IAC 662 (inoculado). El comportamiento del injerto Obatã IAC 1669-20/Robusta (inoculado), posiblemente se deba a un efecto inhibitorio en la translocación de auxina desde la raíz hacia la parte aérea acompañado de un aumento en la translocación de auxinas de la parte aérea hacia la raíz, causado por la presencia de nematodos. ${ }^{14,15} \mathrm{El}$ incremento en el tenor de auxinas, aumentó el crecimiento radicular y consecuentemente la AP. Resultados similares fueron reportados por Rojas \& Salazar. ${ }^{13}$

Por otra parte, Catuaí Amarelo IAC 86/Robusta (no inoculado) y Caturra Vermelho IAC 477/Robusta (no inoculado), estuvieron acordes con el reporte del $\mathrm{IAC}^{30}$, que informa que ambos cultivares son de porte bajo.

El DDT (Figura 2), de acuerdo con Meng et al. ${ }^{31}$, Quintana et $a l .{ }^{32}$ y Ávila et $a l .{ }^{33}$, el DDT puede ser usado como indicador del estatus hídrico y nutricional de las plantas, además, Julca et al. ${ }^{34}$ informan que esta variable forma parte de las características de cada cultivar.

A pesar que Catuaí Vermelho IAC 144/Robusta (inoculado) en la AP no se destacó, en DDT sobresalió en su crecimiento radial alto, probablemente se relacione al alto vigor que presenta este cultivar ${ }^{30}$. En el mismo lugar de estudio, una respuesta similar fue obtenida por Julca et al. ${ }^{18}$ en el cultivar Catuaí. Según Sáenz et al. ${ }^{22}$, el diámetro de tallo se encuentra relacionado con la tasa de sobrevivencia de la planta en campo.

37
El DDT de Obatã IAC 1669-20/Robusta (inoculado) fue consistente con la variable AP, la relación entre ambas variables fue relatada por otros autores como Julca et al. $^{35}$ y Rojas \& Salazar. ${ }^{13}$

Por otra parte, las hojas son tejidos muy importantes, pues ellas llevan a cabo la fotosíntesis. Luego de realizar la comparación de medias, se notó que Caturra Vermelho IAC 477/Robusta (inoculado) mostró mayor NH, mientras que Catuaí Amarelo IAC 86/Robusta (no inoculado) y Limaní/Robusta (inoculado) mostraron menores cantidades de hojas (p $\unlhd 0.05$ ) (Figura 2).

Al ser fuente de fotoasimilados, una menor cantidad de hojas tendría efectos negativos sobre el crecimiento de la planta, notamos que Catuaí Amarelo IAC 86/Robusta (no inoculado) tuvo menor cantidad de tejido foliar, que corresponde marcadamente con los resultados observados en AP y DT (Figura 2).

En general, excepto Obatã IAC 1669-20/Robusta (inoculado), los tratamientos con mayores valores tuvieron ecuaciones de regresión de tipo lineal, además la mayoría de ellos hubo presencia de nematodos. En el caso de los tratamientos con menores valores, todos ellos, salvo el injerto Limaní/Robusta (inoculado), mostraron ecuaciones polinomiales, en general con ausencia de nematodos (Tabla 5). Estos resultados señalan la capacidad que representan estos patógenos al modificar la dinámica de crecimiento de las plantas injertadas de café. Estas modificaciones fueron observadas en otras especies como el melón. ${ }^{36}$

La BS (Figura 3), su relación de la parte aérea, se observó que Laurina IAC 870/Robusta (no inoculado), Caturra Vermelho IAC 477/Robusta (inoculado) y Obatã IAC 1669-20/Robusta (inoculado) fueron significativamente de mayor peso seco. Mien- 
tras que Catuaí Amarelo IAC 86/Robusta (no inoculado) fue menor ( $\mathrm{p} \unlhd 0.05$ ).

En relación a la biomasa en Catuaí Amarelo IAC 86/Robusta (no inoculado), se relaciona a una menor AP y NH, al menor $\mathrm{NH}$ el área fotosintética se ve reducida reflejándose en menor BS de la parte aérea. Los resultados concuerdan con los presentados por Romero et al. ${ }^{37}$

Consistente con el peso seco de la parte aérea, Laurina IAC 870 (no inoculado), Caturra Vermelho IAC 477 (inoculado) y Obatã IAC 1669-20 (inoculado) presentaron mayor masa seca radicular (p $₫ 0.05$ ), mientras que Catuaí Amarelo IAC 86 (no inoculado) obtuvo menor peso seco radicular (Figura 3). Los resultados indican una relación directa entre estas dos variables, además, sugieren el efecto benéfico de 10000 huevos por $\mathrm{kg}$ de substrato en el tenor de biomasa seca de la planta.

En cuanto al IR (Figura 4), Laurina IAC 870/Robusta (no inoculado) y Bourbon Vermelho IAC 662/Robusta (inoculado) fueron menos robustos, mientras que Catuaí Vermelho IAC 99/Robusta (inoculado), Catuaí Vermelho IAC 99/Robusta (no inoculado), Catuaí Amarelo IAC 86/Robusta (no inoculado) y Limaní/Robusta (no inoculado) presentaron mayor robustez. De acuerdo con Sáenz et al. ${ }^{22}$, menores valores en IR indican que la planta tiene más capacidad de sobrevivencia en campo bajo condiciones ambientales no muy favorables para su desarrollo.

En aquellos tratamientos que mostraron un mayor IR, se notó que la mayoría de ellos no fueron inoculados con nematodos. Tomando en cuenta los valores de las variables en forma aislada, estos resultados indican, que morfológicamente Laurina IAC 870/Robusta (no inoculado) y Bourbon Vermelho IAC 662/Robusta (inoculado) pueden ser superiores, sin embargo, esta mejoría no se reflejó en un mayor
IR. Asimismo, sus valores fueron menores a los informados por Julca et al..$^{35}$ y Arizaleta \& Pire. ${ }^{38}$

El ICD, es un importante indicador de desarrollo de plantas en vivero. ${ }^{39}$ Este índice toma en cuenta diferentes variables de crecimiento, por lo cual es muy usado para determinar la calidad de plántulas creciendo en vivero ${ }^{22}$. Catuaí Vermelho IAC 144/Robusta (no inoculado), Caturra Vermelho/Robusta IAC 477 (inoculado) y Obatã IAC 1669-20/Robusta (inoculado) presentaron los valores más altos, mientras que Catuaí Amarelo IAC 86 (no inoculado) y Bourbon Vermelho IAC 662 (no inoculado) tuvieron los valores más bajos ( $\mathrm{p} \unlhd 0.05$ ) (Figura 4).

En un experimento similar, Julca et al. ${ }^{18}$ reportaron que los cultivares Icatú vermelho y Caturra vermelho injertados sobre robusta, en presencia de nematodos, mostraron valores de ICD de 1.79 , cuyo valor fue muy superior obtenido en este ensayo, lo que sugiere en nuestro experimento no hubo un buen balance entre la parte aérea y la raíz. De acuerdo con Olliet ${ }^{40}$, valores altos de ICD indicarían un mejor balance entre el crecimiento de la parte aérea y el crecimiento radicular.

Se agruparon los tratamientos en función de las variables $\mathrm{AP}, \mathrm{DT}, \mathrm{NH}, \mathrm{A} / \mathrm{R}$, una vez realizado el análisis Cluster (Figura 5), notamos que existen básicamente dos grupos: aquellos formados por sus los valores más altos y bajos. El primer grupo estuvo formado mayoritariamente por tratamientos inoculados con nematodos, mientras que el segundo por los tratamientos libres de nematodos. Estos resultados podrían indicar los cultivares de café introducidos responden bien cuando son injertados sobre C. canephora y en presencia de nematodos. Resultados similares fueron reportados por Julca et al. ${ }^{18}$. Evaluadas cada una de las variables, concluimos que tanto el crecimiento como la calidad de las plántulas injertadas sobre $C$. canephora, se vieron 
influenciados por la presencia de nematodos, asimismo, cabe resaltar el comportamiento de los injertos Caturra vermelho IAC 147/Robusta y Obatã IAC 1669-20/Robusta, los cuales mostraron reaccionar bien ante la presencia de Meloidogyne spp.

\section{Conflictos de intereses}

Los autores declaran que no tiene ningún conflicto de interés con esta investigación.

\section{Agradecimientos}

Los autores agradecen a la Junta Nacional del Café (JNC) por financiar la presente investigación, además agradecemos al IRD-Selva de la Universidad Nacional Agraria La Molina, por permitir el uso de sus instalaciones para desarrollar el experimento.

\section{Literatura citada}

1. Instituto Nacional de Estadística e Informática. Evolución de las exportaciones e importaciones. Informe Técnico No 2; febrero 2018. [Consultado 5 mayo 2018]. Disponible en: https://www.inei. gob.pe/media/MenuRecursivo/boletines/02-infor me-tecnico-n02_exportaciones-e-importacionesdic2017.pdf.

2. Instituto Nacional de Innovación Agraria. Situación actual del café en el país. [Consultado 5 mayo 2018]. Disponible en: http://minagri.gob.pe/ portal/485-feria-scaa/10775-el-cafe-peruano.

3. Guevara E, Mestanza C, Oliva M, Vera N. Población de nematodos fitoparasitos asociados al cultivo de café (Coffea arabica L.) en relación a la textura del suelo, Cuipes, Bongará, Amazonas. Rev Indes 2015;1(2):9-18.
4. Barbosa JC, Maldonado-Junior W. AgrostatSistema para análises estatísticas de ensaios agronômicos. Versão 1.0. Joboticabal: Departamento de Ciências Exatas. 2010; Brasil.

5. Saucet S, Ghelder C, Abad P, Duval H, Esmenjaud D. Resistance to root-knot nematodes Meloidogyne spp. in woody plant. New Phytol 2016;211:41-56. DOI: https://doi.org/10.1111/ nph.13933.

6. Barros AF, Oliveira RDL, Zambolim L, Ferreira AO, Coutinho RR. Meloidogyne paranaensis attacking coffee trees in Espirito Santo State, Brazil. Australas Plant Dis Notes 2011;6(1):43-5. DOI: https://doi.org/10.1007/s13314-011-0015-9.

7. Guan W, Zhao X. Root-knot nematode resistance, yield, and fruit quality of specialty melons grafted onto Cucumis metulifer. HortScience 2014;49(8):1049-51.

8. Fatobene B, Andrade VT, Gonçalves W, Guerreiro-Filho O. Coffea canephora clones with multiple resistance to Meloidogyne incognita and $M$. paranaensis. Exp Agric 2018:1-9. DOI: https:// doi.org /10.1017/S0014479718000108.

9. Andreazi E, Sera G, Faria R, Sera T, Fonseca I, Machado A, et al. Behavior of 'IPR100' and 'Apoatã IAC 2258' coffee cultivars under different infestation levels of Meloidogyne paranaensis inoculum. Aust J Crop Sci 2015;9(11):1069-74.

10.Carvalho J, Carvalho G, Ferreira A, Rezende J, Dias R, Carvalho A. Dinâmica de macro nutrientes em genótipos de Coffea canephora com potencial para utilização como porta-enxerto. Coffee Science 2014;10(2):176-83.

11.Tomaz M, Sakiyama N, Matta F, Martínez H, Cruz C, Pereira A. Efeito do porta-enxerto nas trocas gaseosas, área foliar e superficie de raíz de mudas de Coffea arabica L. CERES 2006;53(306):237-42. 
12.Ferrari R, Sakiyama N, Tomaz M, Cruz C, Pereira A. Análise dialélica de cafeeiros enxertados, em condições de campo. CERES 2005;52(300): 165-75.

13.Rojas M, Salazar, L. Densidad crítica de Meloidogyne exígua en plantas de almácigos de café variedad Caturra. Agro Costarricense 2013;37(2): 115-23.

14.Curtis R. Do phytohormones influence nematode invasion and feeding site establishment? Nematology 2007;9(2):155-60. DOI: https://doi.org/10. $1163 / 156854107780739072$.

15.Kyndt T, Haegeman A, Warmerdan S, Wanjau C, Jahani M, Engler G, et al. Redirection of auxin flow in Arabidopsis thaliana roots after infection by root-knot nematodes. J Exp Bot 2016;67(15):4559-70. DOI: https://doi.org/10. 1093/jxb /erw230.

16.Goreta S, Žanić K, Dumičić G, Raspudić E, Vuletin G, Ban D. Growth and yield of grafted cucumbers in soil infested with root-knot nematodes. Chilean JAR 2014;74(1): 29-34. DOI: https://doi.org/10.4067/S0718-583920140001000 $\underline{05}$.

17. Barbosa D, Vieira H, Rodrigues W, Rodrigues J, Barroso D, Silva T. Efeito da enxertia e do nematoide Meloidogyne exigua sobre o crescimento e a produtividade de cafeeiros. Coffee Science 2014;9(4):427-34.

18.Julca-Otiniano A, Andía-Alarcón E, EstelitaCastro S, Borjas-Ventura R. Comportamiento de Coffea arabica L. injertadas sobre Coffea canephora en presencia de nematodos en vivero. Rev Investig Altoandin 2018;20(3):267-80. DOI: http://dx.doi.org/ 10.18271/ria.2018.391.

19.Julca-Otiniano A, Blas-Sevillano R, CruzJanampa R, Bello-Amez S, Borjas-Ventura R, Talaverano-Tambraico D, et al. Introducción de variedades de café de Brasil. Ed. Universidad Nacional Agraria La Molina; 2011. Lima, Perú.

20.Julca-Otiniano A, Meneses-Florián L, BelloAmez S, Borjas-Ventura R, Anahui-Andia J, Talaverano-Tambraico D, et al. Selección de fuentes naturales para la fertilización de café en el marco de una agricultura orgánica. Universidad Nacional Agraria La Molina; 2009. Lima. Perú. p. 59.

21.Asociación Nacional del Café. Evaluación de tres nematicidas y la práctica de injerto hipocotiledonar en el control de Pratylenchus sp. [Consultado 31 mayo 2017]. Disponible en https://www .anacafe.org/glifos/index.php?title=Injerto_Reyn a_Pratylenchus_SP.

22.Sáenz R, Muñoz F, Villaseñor R, , Prieto R, Rueda S. Calidad de plantas en viveros forestales de clima templado en Michoacán. Folleto técnico No 17. SAGARPA-INIFAP-CIRPAC Campo Experimental Uruapan. Uruapan, Mich. 2010; México.

23. Villar P. Importancia de la calidad de planta en los proyectos de revegetación. En: Restauración de Ecosistemas Mediterráneos. Universidad de Alcalá/ Asociación Española de Ecología Terrestre. España; 2003.

24.Dickson A, Hosner F. Quality appraisal of white spruce and white pine seedling stock in nursery. Forest Chron 1960;36:10-3. DOI: https://doi.org/ 10. 5558/tfc36010-1.

25.Montgomery D. Diseño y análisis de experimentos. 2da Edición. Editorial LIMUSA. México; 2005.

26. Mukthar T, Hussain M, Kayani M. Yield responses of 12 okra cultivars to southern root-knot nematode (Meloidogyne incognita). Bragantia 2017;76(1):108-12.

27.Kayani M, Mukhtar T, Hussain M. Interaction between nematode inoculum density and plant 
age on growth and yield of cucumber and reproduction of Meloidogyne incognita. Pak J Zool 2018;50(3):897-902. https://doi.org/10.17582/jou rnal.pjz/2018.50.3.897.902.

28.Ko D, Kang J, Kiba T, Park J, Kojima M, Do J, et al. Arabidopsis ABCG14 is essential for the root-shoot translocation of cytokinin. Proc Natl Acad Sci USA 2014;111(19):7150-5. DOI: https: //doi. org/10.1073/pnas.1321519111.

29. Gratão PL, Monteiro CC, Tezotto T, Carvalho RF, Alves LR, Peters LP, et al. Cadmium stress antioxidant response and root-shoot comunication in grafted tomato plants. Biometals 2015;28:803-16. DOI: https://doi.org/10.1007/s1 0534-015-9867-3.

30.Instituto Agronómico de Campinas. Cultivares de café desenvolvidas pelo Instituto Agronómico (IAC) e registrada no Ministério da Agricultura Pecuaria e Abastecimento; 2017. [Consultado 30 mayo 2017]. Disponible en http://www.iac.sp. gov.br/areasdepesquisa/cafe/tabela_rnc_cultivare s_cafe_iac.pdf.

31.Meng Z, Duan A, Dassanayake K, Wang X, Liu $\mathrm{Z}$, Liu $\mathrm{H}$, et al. Suitable indicators using stem diameter variation-derived indices to monitor the water status of greenhouse tomato plants. PloS One 2017;12(2):e0171423. DOI: https://doi.org /10. 1371/journal.pone.0171423.

32. Quintana-Escobar AO, Iracheta-Donjuan L, Méndez-López I, Alonso-Báez M. Caracterización de genótipos elite de Coffea canephora por su tolerancia a la sequía. Agro Mesoam 2017;28(1): 183-98. DOI: https://doi:10.15517/ am.v28 i1.23874.

33.Ávila-Peralta O, Mendoza-Villarreal R, ValdezAguilar LA, Rodríguez-Campos EM, HernándezPérez A, Cárdenas-Flores A. Crecimiento y estado nutricional de tomate en respuesta a sustratos orgánicos y hongos micorrízicos. Rev Mex Cienc Agrícolas 2015; 12: 2409-2422.

34.Julca A, Echevarría C, Janampa R, Borjas R, Bello S. Comparativo en vivero de cinco variedades de café (Coffea arabica L.) en San Ramón, Chanchamayo. JISTH 2015b; 56: 86-90.

35.Julca-Otiniano A, Borjas-Ventura R, Bello-Amez S, Ladera-Manyari Y, Rebaza-Fernández D. El crecimiento del café var. Caturra Roja y su relación con la aplicación de abonos orgánicos. Saber y Hacer 2015a; 2(2): 75-89.

36.Kayani MZ, Mukhtar T, Hussain MA. Effect of southern root knot nematode population densities and plant age on growth and yield parameters of cucumber. Crop Prot 2017;92:207-12. DOI: http: //dx. doi.org/10.1016/j.cropro.2016.09.007.

37.Romero-Munar A, Del-Saz NF, Ribas-Carbó M, Flexas J, Baraza E, Florez-Sarasa I, et al. Arbuscular mycorrhyzal symbiosis with Arundo donax decreases root respiration and increases both photosynthesis and plant biomass accumulation. Plant Cell Environ 2017;40(7):1115-26. DOI: https:// doi.org/10.1111/pce.12902.

38. Arizaleta M, Pire R. Respuestas de plántulas de cafeto al tamaño de la bolsa y fertilización con nitrógeno y fósforo en vivero. Agrociencia 2008;42(1):47-55.

39.Basave-Villalobos E, García-Castillo LC, CastroRíos A, Calixto-Valencia CG, Sigala-Rodriguez JA, García-Pérez JL. Plant Quality of Cedrela odorata L. associated with nursery cultural practices. Rev Mexi Cienc Forestales 2016;7(36):6580.

40.Olliet J. La calidad de la postura forestal en vivero. Escuela Técnica Superior de Ingenieros Agronómos y de Montes de Córdoba. España; 2000. 